\title{
A NOTE ON CARLESON MEASURES IN PRODUCT SPACES
}

\author{
R. FEFFERMAN
}

\begin{abstract}
In this article we give a very simple proof of a basic theorem about the class BMO in the multiple parameter setting.
\end{abstract}

The purpose of this note is to give a very simple proof of the following result.

THEOREM. Suppose $f \in L^{\infty}\left(\mathbf{R}^{2}\right)$ and let $u$ denote its biharmonic extension to $\mathbf{R}_{+}^{2} \times \mathbf{R}_{+}^{2}$. Then $\left|\nabla_{1} \nabla_{2} u\right|^{2} y_{1} y_{2} d z_{1} d z_{2}$ is a Carleson measure on $\mathbf{R}_{+}^{2} \times \mathbf{R}_{+}^{2}$.

For the definition of Carleson measure, as well as notation which we use here, see Chang [1] and Chang-Fefferman [2].

The theorem above is due to Alice Chang [1]. Somewhat later alternative proofs were found by R. Fefferman $[\mathbf{3}]$ and E. M. Stein [4]. Stein's proof is already quite short and is based on identities for harmonic functions and Green's theorem, while ours is real variable in nature. What follows is the result of discussions and work with Alice Chang, R. R. Coifman, and Y. Meyer, and the author wishes to thank them.

Our proof is based on the following simple lemma:

LEMMA. Let $\psi_{i}(x)$ for $i=1,2$ be a function on $\mathbf{R}^{1}$ supported in $|x|<1$ with $\left\|\psi_{i}\right\|_{1}=1, \psi_{i} \in L^{2}$, and $\int \psi_{i}=0$. Let $\Omega \subseteq \mathbf{R}^{2}$ be an open set of finite measure, and define

$$
\Psi_{y_{1}, y_{2}}\left(x_{1}, x_{2}\right)=y_{1}^{-1} y_{2}^{-1} \psi_{1}\left(x_{1} / y_{1}\right) \psi_{2}\left(x_{2} / y_{2}\right)
$$

Finally, set

$$
\mathcal{L}_{\Psi}(f)=\left(\iint_{S(\Omega)}\left|f * \Psi_{y_{1}, y_{2}}\right|^{2} \frac{d t d y}{y_{1} y_{2}}\right)^{1 / 2}
$$

Then

$$
\mathcal{L}_{\Psi}(f) \leq C\left(\prod_{i=1}^{2} \log \left(\left\|\psi_{i}\right\|\right)_{2}\right)^{1 / 2} \cdot\|f\|_{\infty}|\Omega|^{1 / 2} .
$$
then

Proof of THE Lemma. When $\left(t_{1}, t_{2}, y_{1}, y_{2}\right)$ is in the Carleson region $S(\Omega)$,

$$
f * \Psi_{y_{1}, y_{2}}(t)=f_{0} * \Psi_{y_{1}, y_{2}}(t), \quad \text { where } f_{0}=f \chi_{\Omega} .
$$

This gives

$$
\mathcal{L}_{\Psi}(f) \leq\left(\iint_{\mathbf{R}_{+}^{2} \times \mathbf{R}_{+}^{2}}\left|f_{0} * \Psi_{y_{1}, y_{2}}\left(t_{1}, t_{2}\right)\right|^{2} \frac{d t d y}{y_{1} y_{2}}\right)^{1 / 2}
$$

Received by the editors May 4, 1984, and, in revised form, June 18, 1984.

1980 Mathematics Subject Classification. Primary 42A18.

Key words and phrases. Hardy spaces, duality, biharmonic. 
The usual argument with the Plancherel theorem shows this last expression to be

$$
\begin{aligned}
& \leq\left\|f_{0}\right\|_{2}\left(\prod_{i=1}^{2} \int_{\mathbf{R}^{1}}\left|\hat{\psi}_{i}(\xi)\right|^{2} \frac{d \xi}{|\xi|}\right)^{1 / 2} \\
& \leq\|f\|_{\infty}|\Omega|^{1 / 2}\left(\prod_{i=1}^{2} \int_{\mathbf{R}^{1}}\left|\hat{\psi}_{i}(\xi)\right|^{2} \frac{d \xi}{|\xi|}\right)^{1 / 2} .
\end{aligned}
$$

But for $|\xi|<1,\left|\hat{\psi}_{i}(\xi)\right| \leq|\xi|$ since $\hat{\psi}_{i}(0)=0$ and $\left|\hat{\psi}_{i}^{\prime}(\xi)\right|=\left|\left[x \psi_{i}(x)\right]^{\wedge}(\xi)\right| \leq 1$. This gives

$$
\int_{|\xi| \leq 1}\left|\hat{\psi}_{i}(\xi)\right|^{2} \frac{d \xi}{|\xi|} \leq 1
$$

Obviously, $\left|\hat{\psi}_{i}(\xi)\right| \leq 1$, so

$$
\int_{1<|\xi|<\left\|\psi_{i}\right\|_{2}^{2}}\left|\hat{\psi}_{i}(\xi)\right|^{2} \frac{d \xi}{|\xi|} \leq c \log \left\|\psi_{i}\right\|_{2} .
$$

Finally,

$$
\int_{|\xi|>\left\|\psi_{i}\right\|_{2}^{2}}\left|\hat{\psi}_{i}(\xi)\right|^{2} \frac{d \xi}{|\xi|} \leq \frac{1}{\left\|\psi_{i}\right\|_{2}^{2}} \int_{\mathbf{R}^{\prime}}\left|\hat{\psi}_{i}(\xi)\right|^{2} d \xi=1 .
$$

This concludes the proof of the Lemma.

PROOF OF THE THEOREM. We prove that for open sets $\Omega$,

$$
\iint_{S(\Omega)}\left|\frac{\partial^{2} u}{\partial y_{1} \partial y_{2}}\right|^{2} y_{1} y_{2} d z_{1} d z_{2} \leq C|\Omega| \text {. }
$$

(The part of $\nabla_{1} \nabla_{2}$ coming from $\partial^{2} u / \partial y_{1} \partial y_{2}$, etc., is handled similarly.) This amounts to estimating

$$
\left(\iint_{S(\Omega)}\left|f * \Psi_{y_{1}, y_{2}}\left(t_{1}, t_{2}\right)\right|^{2} \frac{d t d y}{y_{1} y_{2}}\right)^{1 / 2}=\mathcal{L}_{\Psi}(f)
$$

for $\Psi\left(x_{1}, x_{2}\right)=\psi\left(x_{1}\right) \psi\left(x_{2}\right)$, where

$$
\psi(x)=\frac{1-x^{2}}{1+x^{2}} \cdot \frac{1}{1+x^{2}} .
$$

For $k \geq 1$ let

$$
\psi_{k}(x)=\psi(x)\left[\chi_{2^{k-1} \leq|x|<2^{k}}(x)-c_{k} \chi|x| \leq 1(x)\right],
$$

where $c_{k}$ is chosen so that $\int \psi_{k}=0$. Then $c_{k}=O\left(2^{-k}\right)$ and $\sum \psi_{k}=\psi$. For integers $k, j \geq 1$, set $\Psi_{k, j}\left(x_{1}, x_{2}\right)=\psi_{k}\left(x_{1}\right) \psi_{j}\left(x_{2}\right)$. Let $\tilde{\Omega}_{k, j}=\left\{M_{s}\left(\chi_{\Omega}\right)>1 / 2^{k+j}\right\}$. Then, applying the argument of the Lemma with $\Psi_{k, j}$ replacing $\Psi$ and $f \chi_{\tilde{\Omega}_{k, j}}$ replacing $f \chi_{\Omega}$, we get

$$
(\sim) \mathcal{L}_{\Psi_{k, j}}(f) \leq C\left[\log 2^{k} \log 2^{j}\right]^{1 / 2} \cdot 2^{-(k+j)}\|f\|_{\infty}\left|\tilde{\Omega}_{k, j}\right|^{1 / 2}
$$

By the strong maximal theorem $\left|\tilde{\Omega}_{k, j}\right| \leq C 2^{k+j}(k+j)|\Omega|$, and Minkowski's inequality gives $\mathcal{L}_{\Psi}(f) \leq \sum_{j, k} \mathcal{L}_{\Psi_{k, j}}(f)$. Taking the estimate of $\left|\tilde{\Omega}_{k, j}\right|$ into account and summing $(\sim)$ on $k, j$ completes the proof. 


\section{REFERENCES}

1. S. Y. Chang, Carleson measure on the bi-disc, Ann. of Math. (2) 109 (1979).

2. S. Y. Chang and R. Fefferman, A continuous version of the duality of $H^{1}$ and $B M O$ on the bi-disc, Ann. of Math. (2) 112 (1980).

3. R. Fefferman, Functions of bounded mean oscillation on the bi-disc, Ann. of Math. (2) 110 (1979).

4. E. M. Stein, A variant of the area integral, Bull. Sci. Math. (2) 103 (1979).

Department of Mathematics, University of Chicago, Chicago, Illinois 60637 